\title{
ISOMETRIES IN SEMISIMPLE, COMMUTATIVE BANACH ALGEBRAS
}

\author{
KRZYSZTOF JAROSZ
}

\begin{abstract}
We show that for any semisimple, commutative, complex Banach algebra $A$ with unit there are norms on $A$, which we call natural norms, equivalent to the original norm on $A$ with the following property: Let $\left(A,\|\cdot\| \|_{A}, e_{A}\right)$ and $\left(B,\|\cdot\|_{B}, e_{B}\right)$ are commutative, semisimple Banach algebras with units and natural norms. Assume $T$ is a linear isometry from $\left(A,\|\cdot\|_{A}\right)$ onto $\left(B,\|\cdot\|_{B}\right)$ with $T e_{A}=e_{B}$. Then $T$ is an isomorphism in the category of Banach algebras. For a fairly large class of algebras, for example, for uniform algebras, for algebras of the form $C^{k}(X), \operatorname{Lip}(X), \operatorname{AC}(X)$, the natural norm we have defined coincides with a usual norm.
\end{abstract}

0. Introduction. Let $A$ and $B$ be uniform algebras, i.e., Banach algebras with units such that $\left\|f^{2}\right\|=\|f\|^{2}$ for all $f \in A$ (for all $f \in B$, respectively). The wellknown Nagasawa theorem [4] states that $A$ and $B$ are isometric if and only if they are isomorphic in the category of algebras, and that every linear isometry from $A$ onto $B$ which preserves units is an isomorphism of algebras. In $[1,2,5-7]$ it has been proved that the Nagasawa theorem remains true for some other Banach algebras.

In $1965 \mathrm{M}$. Cambern [1] proved that if both $A$ and $B$ are equal to $C^{1}[0,1]$ or to $\mathrm{AC}[0,1]$, then any isometry of $A$ onto $B$ is induced by a homeomorphism of the unit interval $[0,1]$. Here $C^{1}[0,1]$ is the algebra of complex-valued, continuously differentiable functions on $[0,1]$ with norm

$$
\|f\|=\max _{0 \leq t \leq 1}\left(|f(t)|+\left|f^{\prime}(t)\right|\right) \quad \text { for } f \in C^{1}[0,1],
$$

and $\mathrm{AC}[0,1]$ is an algebra of complex-valued, absolutely continuous functions on $[0,1]$ with norm

$$
\begin{aligned}
\|f\| & =\|f\|_{\infty}+\left\|f^{\prime}\right\|_{1} \\
& =\max _{0 \leq t \leq 1}|f(t)|+\int_{0}^{1}\left|f^{\prime}(t)\right| d t \quad \text { for } f \in \operatorname{AC}[0,1] .
\end{aligned}
$$

In 1971 N. V. Rao and A. K. Roy [7] proved that this holds for algebras of Lipschitz functions and continuously differentiable functions both with norm $\|f\|=$ $\|f\|_{\infty}+\left\|f^{\prime}\right\|_{\infty}$.

In 1981 M. Cambern and V. Pathak [2] proved it for $C_{0}^{1}(X)$, where $X$ is a closed subset of the real line containing no isolated points. Here $C_{0}^{1}(X)$ is a Banach space of continuously differentiable functions $f$ on $X$ which are such that $f$ and $f^{\prime}$ are zero at infinity with norm defined as for $C^{1}[0,1]$.

Received by the editors January 9, 1984 and, in revised form, April 23, 1984.

1980 Mathematics Subject Classification. Primary 46J05; Secondary 46B20, 46B25.

(C) 1985 American Mathematical Society $0002-9939 / 85 \$ 1.00+\$ .25$ per page 
Recently, Pathak extended the above result to $\mathrm{AC}(X)$, the space of absolutely continuous functions on a closed subset $X$ of the real line with norm $\|f\|=\|f\|_{\infty}+$ variation of $f$ on $X[6]$, and to $C^{(n)}[0,1]$, the Banach algebra of complex-valued, $n$-times continuously differentiable functions on $[0,1]$ with norm

$$
\|f\|=\sup _{t \in[0,1]}\left(\sum_{r=0}^{n}\left(\frac{\left|f^{(r)}(t)\right|}{r !}\right)\right) .
$$

Whether the Nagasawa theorem holds for some Banach algebras $A$ and $B$ depends not only on the algebraic structure of $A$ and $B$, but also, and in fact mostly, on the norms in these algebras. For any Banach algebra $A$ there are a number of equivalent, submultiplicative norms on $A$. Very simple examples (e.g., $l^{1}$ with convolution multiplication) show that the Nagasawa theorem does not hold in general for semisimple, commutative Banach algebras with unit. In this paper we define natural norms on these algebras, we show that they possess a number of natural norms, and we prove that the Nagasawa theorem holds for such an algebra if it is equipped with a natural norm.

The norms on $C^{1}[0,1], C_{0}^{1}(X), \operatorname{AC}(X)$, and $\operatorname{Lip}[0,1]$ of the forms $\|f\|=\|f\|_{\infty}+$ $\left\|f^{\prime}\right\|_{1},\|f\|=\|f\|_{\infty}+\left\|f^{\prime}\right\|_{\infty}$, or $\|f\|=\|f\|_{\infty}+$ variation of $f$ are natural, but the Cambern-Pathak norm on $C^{(n)}[0,1]$ is not; nevertheless there are natural norms on $C^{(n)}[0,1]$, for example, $\|f\|=\|f\|_{\infty}+\left\|f^{\prime}\right\|_{\infty}+\cdots+\left\|f^{(n)}\right\|$.

All of these algebras consist of functions defined on a subset of the real line. There are analogous algebras defined on a subset of Euclidean $n$-space if we define, in such an algebra, a norm by $\|f\|=\|f\|_{\infty}+\left\|D_{f}\right\|^{\prime}$, where $D_{f}$ denotes the differential of $f$ and $\|\cdot\|^{\prime}$ is a seminorm. We derive other examples of natural algebras.

The idea of the proof is very simple. We only use, roughly speaking, the fact that a natural norm on $A$ is a combination of the usual sup-norm and a seminorm $\|\cdot\|^{\prime}$ such that $\left\|e_{A}\right\|^{\prime}=0$.

Our results also hold for some normed spaces which are not algebras.

1. Definitions and notations. We denote by $P$ the set of all norms on the two-dimensional real linear space with $p((1,0))=1$.

For $p \in P$ we put

$$
D(p)=\lim _{t \rightarrow 0^{+}} \frac{p(1, t)-1}{t} .
$$

For $z_{0} \in \mathbf{C}$ and $r \geq 0$ we put

$$
K\left(z_{0}, r\right)=\left\{z \in \mathbf{C}:\left|z-z_{0}\right| \leq r\right\}
$$

and write $K(r)$ in place of $K(0, r)$.

If $K, H$ are subsets of the complex plane $\mathbf{C}$ we denote by $\operatorname{co}(K)$ the convex hull of $K$, we put

$$
K+H=\{w+z: w \in K, z \in H\}
$$

and, for $z_{0} \in K$,

$$
\begin{aligned}
\rho\left(K, z_{0}\right) & =\sup \left\{r \geq 0: \exists z \in K z_{0} \in K(z, r) \subset K\right\}, \\
\rho(K) & =\inf \{\rho(K, z): z \in K\} .
\end{aligned}
$$

Assume $X$ is a compact, Hausdorff space, $A$ is a linear subspace of $C(X)$, and $A$ contains the function constantly equal to one, which we denote by 1 . Then: 
By $\|\cdot\|_{\infty}$ we denote the usual sup-norm on $A$.

We call a seminorm $\|\cdot\|$ on $A$ one-invariant if $\|a+\mathbf{1}\|=\|a\|$ for all $a \in A$.

Let $p \in P$ and let $\|\cdot\|$ be a norm on $A$; then we call it $p$-norm if there is a one-invariant seminorm $\|\cdot \mid\|$ on $A$ such that $\|\cdot\|=p\left(\|\cdot\|_{\infty},\|\| \cdot\|\|\right)$.

We call $\|\cdot\|$ a natural norm on $A$ if it is a $p$-norm for some $p \in P$.

By $\bar{A}$ we denote the closure of $A$ in $\left(C(X),\|\cdot\|_{\infty}\right)$.

By $\mathrm{Ch} A$ we denote the set of extreme points $F$ of the unit ball of $\left(A,\|\cdot\|_{\infty}\right)^{*}$ such that $F(\mathbf{1})=1$, and we identify $\mathrm{Ch} A$ with a subset of $X$.

For $f \in A$ we put $\sigma(f)=f(X)$ and $\tilde{\sigma}(f)=\operatorname{co}(f(X))$.

We call $A$ a regular subspace of $C(X)$ if for any $\varepsilon>0$, any $x_{0} \in \mathrm{Ch} A$, and any open neighborhood $U$ of $x_{0}$ there is an $f \in A$ with $\|f\|_{\infty} \leq 1+\varepsilon, f\left(x_{0}\right)=1$, and $|f(x)|<\varepsilon$ for $x \in X \backslash U$.

In the sequel any semisimple, commutative Banach algebra $A$ is identified, via the Gelfand transformations [3], with a subalgebra of $C(\mathfrak{M}(A))$, with $\mathfrak{M}(A)$ being the maximal ideal space of $A$.

\section{The results.}

THEOREM. Let $X$ and $Y$ be compact Hausdorff spaces, let $A$ and $B$ be complex linear subspaces of $C(X)$ and $C(Y)$, respectively, and let $p, q \in P$. Assume $A$ and $B$ contain constant functions, and let $\|\cdot\|_{A},\|\cdot\|_{B}$ be a p-norm and q-norm on $A$ and $B$, respectively. Assume next that there is a linear isometry $T$ from $\left(A,\|\cdot\|_{A}\right)$ onto $\left(B,\|\cdot\|_{B}\right)$ with $T \mathbf{1}=\mathbf{1}$. Then if $D(p)=D(q)=0$, or if $A$ and $B$ are regular subspaces of $C(X)$ and $C(Y)$, respectively, then $T$ is an isometry from $\left(A,\|\cdot\|_{\infty}\right)$ onto $\left(B,\|\cdot\|_{\infty}\right)$.

Before proving the theorem let us prove two propositions which show that any semisimple, commutative Banach algebra $A$ with unit possesses natural norms and is a regular subspace of $C(\mathfrak{M}(A))$.

Proposition 1. Let $(A,\|\cdot\|, \mathbf{1})$ be a commutative, semisimple Banach algebra with unit. Then for any $p \in \mathcal{P}$ there is a p-norm $\|\cdot\|_{A}$ on $A$ which is submultiplicative and equivalent to the original one.

Proof. Fix $x_{0} \in \mathfrak{M}(A)$, put $A_{0}=\left\{a \in A: a\left(x_{0}\right)=0\right\}$, and let $P$ be a linear, continuous projection from $A$ onto $A_{0}$ with $P \mathbf{1}=0$. Fix a positive integer $k$ and define

$$
\|a\|_{A}=p\left(\|a\|_{\infty}, k\|P a\|\right) \text { for } a \in A .
$$

It is easy to check that $\|\cdot\|_{A}$ is a $p$-norm on $A$, equivalent to the original one, and if $k$ is sufficiently large then $\|\cdot\|_{A}$ is submultiplicative.

Proposition 2. Let $(A,\|\cdot\|, \mathbf{1})$ be a commutative, semisimple Banach algebra with unit. Then $A$ is a regular subspace of $C(\mathfrak{M}(A))$.

Proof. Fix an $x_{0} \in \operatorname{Ch} A .\left(\bar{A},\|\cdot\|_{\infty}\right)$ is a function algebra with Choquet boundary equal to $\mathrm{Ch} A$, so $[3]$ there is a net $\left(f_{\alpha}\right)_{\alpha \in \Gamma} \subset \bar{A}$ such that $\left\|f_{\alpha}\right\|=$ $1=f\left(x_{0}\right)$, and $\left(f_{\alpha}\right)$ tends uniformly to zero off any neighbourhood of $x_{0}$. Since $\left(A,\|\cdot\|_{\infty}\right)$ is a dense subset of $\left(\bar{A},\|\cdot\|_{\infty}\right)$, for any $\varepsilon>0$ there is an $\tilde{f}_{\alpha} \in A$ with $\left\|\tilde{f}_{\alpha}-f_{\alpha}\right\|_{\infty}<\varepsilon$, and this completes the proof. 
ProOF OF THE THEOREM. For any convex subset $K$ of the complex plane and any $\varphi \in[0,2 \pi)$ we put

$$
c(K, \varphi)=\sup \left\{a \in R: \text { there is a } b \in \mathbf{R} \text { with } a e^{i \varphi}+b e^{i \varphi+i \pi / 2} \in K\right\} .
$$

For any subspace $A$ of $C(X)$ we define the functions:

$$
\begin{aligned}
c_{A}: A \times[0,2 \pi) & \rightarrow \mathbf{R} \quad \text { by } c_{A}(f, \varphi)=c(\tilde{\sigma}(f), \varphi), \\
r_{A}: A \times \mathbf{R}^{+} \times[0,2 \pi) & \rightarrow \mathbf{R}^{+} \quad \text { by } r_{A}(f, t, \varphi)=\left\|f+e^{i \varphi} t \mathbf{1}\right\|_{\infty} .
\end{aligned}
$$

For any $\varphi \in[0,2 \pi), f \in A, t \in \mathbf{R}^{+}$we have

$$
t+c_{A}(f, \varphi) \leq r_{A}(f, t, \dot{\varphi}) \leq \sqrt{\left(t+c_{A}(f, \varphi)\right)^{2}+\|f\|_{\infty}^{2}},
$$

and, hence,

$$
\lim _{t \rightarrow+\infty}\left(r_{A}(f, t, \varphi)-t\right)=c_{A}(f, \varphi)
$$

Let

$$
\|\cdot\|_{A}=p\left(\|\cdot\|_{\infty},\|\| \cdot\|\|_{A}\right), \quad\|\cdot\|_{B}=q\left(\|\cdot\|_{\infty},\|\| \cdot\|\|_{B}\right),
$$

where $\||\cdot|\|_{A}$ and $\||\cdot|\|_{B}$ are one-invariant seminorms on $A$ and $B$, respectively.

Fix $f \in A$ and put $c_{1}=\|\| f\left|\left\|_{A}, c_{2}=\right\|\right| T f \|_{B}$. By our assumption for any positive $t$ and any $\varphi \in[0,2 \pi)$, we have

$$
p\left(r_{A}(f, t, \varphi), c_{1}\right)=q\left(r_{B}(T f, t, \varphi), c_{2}\right) .
$$

Hence, from (1) we get

$$
\begin{aligned}
0= & \lim _{t \rightarrow+\infty}\left[p\left(r_{A}(f, t, \varphi), c_{1}\right)-q\left(r_{B}(T f, t, \varphi), c_{2}\right)\right] \\
= & \lim _{t \rightarrow+\infty}\left[r_{A}(f, t, \varphi) p\left(1, c_{1} / r_{A}(f, t, \varphi)\right)-r_{A}(f, t, \varphi)\right] \\
& +\lim _{t \rightarrow+\infty}\left[r_{B}(T f, t, \varphi)-r_{B}(T f, t, \varphi) q\left(1, c_{2} / r_{B}(T f, t, \varphi)\right)\right] \\
& +\lim _{t \rightarrow+\infty}\left(r_{A}(f, t, \varphi)-r_{B}(T f, t, \varphi)\right) \\
= & c_{1} D(p)-c_{2} D(q)+c_{A}(f, \varphi)-c_{B}(T f, \varphi)
\end{aligned}
$$

Hence,

(2)

$c_{B}(T f, \varphi)-c_{A}(f, \varphi)=\|\| f\|\|_{A} D(p)-\||| T f \mid\|_{B} D(q)$ for all $f \in A$ and $\varphi \in[0,2 \pi)$.

LEMMA 1. Let $K$ and $H$ be compact, convex subsets of the complex plane. Assume $c(K, \varphi)=c(H, \varphi)$ for all $\varphi \in[0,2 \pi)$. Then $K=H$.

ProOF. Assuming the contrary, we obtain that there is a $z_{0} \in H \backslash K$. Since $K$ is convex and compact, there is a line $l$ such that $\mathbf{C} \backslash l$ is a sum of two connected components $\mathbf{C}^{+}$and $\mathbf{C}^{-}$with $K \subset \mathbf{C}^{+}$and $z_{0} \in \mathbf{C}^{-}$. Let $\varphi_{0} \in[0,2 \pi)$ be such that the vector $\left(\cos \varphi_{0}, \sin \varphi_{0}\right)$ is orthogonal to $l$ and has direction from $\mathbf{C}^{+}$to $\mathbf{C}^{-}$. We have

$$
c\left(K, \varphi_{0}\right)<c\left(\mathbf{C}^{+}, \varphi_{0}\right)<c\left(\left\{z_{0}\right\}, \varphi_{0}\right) \leq c\left(H, \varphi_{0}\right) .
$$

For any $f \in A$ set $\Delta f=\|\| f\left|\left\|\left.\right|_{A} D(p)-\right\|\right||T f| \|_{B} D(q)$. For any $r \geq 0$ and any compact convex subset $K$ of $\mathbf{C}$, we have

$$
c(K+K(r), \varphi)=c(K, \varphi)+r \quad \text { for all } \varphi \in[0,2 \pi) .
$$


Hence, by (2) and the lemma for any $f \in A$, we get:

$$
\begin{aligned}
& \text { if } \Delta f \geq 0 \text { then } \tilde{\sigma}(T f)=\tilde{\sigma}(f)+K(\Delta f) ; \\
& \text { if } \Delta f \leq 0 \text { then } \tilde{\sigma}(f)=\tilde{\sigma}(T f)+K(-\Delta f) ;
\end{aligned}
$$

hence,

$$
\|T f\|_{\infty}-\|f\|_{\infty}=\Delta f .
$$

Assume $D(p)=D(q)=0$. Then for any $f \in A$ we have $\Delta f=0$, so (4) gives the result.

Assume now $A$ and $B$ are regular subspaces of $C(X)$ and $C(Y)$, respectively. To finish the proof, by symmetry, it is sufficient to show that

$$
\|T f\|_{\infty}-\|f\|_{\infty}=\Delta f \geq 0 \text { for all } f \in A .
$$

For this we put, for any $\varepsilon>0$,

$$
A_{\varepsilon}=\{f \in A: \rho(\tilde{\sigma}(f)) \leq \varepsilon\} .
$$

We prove (5) by showing the following statements:

1. $T$ is a continuous map from $\left(A,\|\cdot\|_{\infty}\right)$ onto $\left(B,\|\cdot\|_{\infty}\right)$.

2. For each $\varepsilon>0$ the set $A_{\varepsilon}$ is dense in $\left(A,\|\cdot\|_{\infty}\right)$.

3. For each $\varepsilon>0$ and each $f \in A_{\varepsilon}$ we have $\|T f\|_{\infty} \geq\|f\|_{\infty}-\varepsilon$.

Proof of the first step.

LEMMA 2. Assume $A$ is a regular subspace of $C(X)$ with $\mathbf{1} \in A$ and let $x_{0} \in$ $\mathrm{Ch} A$. Then for any $\varepsilon>0$ and any open neighbourhood $U$ of $x_{0}$, there is an $f \in A$ such that

$$
\|f\|_{\infty} \leq 1+\varepsilon, f\left(x_{0}\right)=1,|f(x)+1| \leq \varepsilon \text { for } x \in X \backslash U,
$$

and $|\operatorname{Im} f(x)| \leq \varepsilon$ for all $x \in X$.

Proof. We define, by induction, a sequence $\left(f_{k}\right)_{k=1}^{\infty} \subset A$ and a descending sequence of neighbourhoods $\left(U_{k}\right)_{k=1}^{\infty}$ of the point $x_{0}$ :

We set $U_{1}=U$ and let $f_{1}$ be any function from $A$ such that $\left\|f_{1}\right\|_{\infty} \leq 1+\varepsilon / 2$, $f_{1}\left(x_{0}\right)=1$, and $\left|f_{1}(x)\right|<\varepsilon / 2$ for all $x$ in $X \backslash U_{1}$.

Assume we have defined $U_{k}$ and $f_{k}$. Then

$$
\begin{aligned}
& U_{k+1}=\left\{x \in U_{k}:\left|f_{k}(x)-1\right|<\varepsilon / 2\right\} \\
& \left\|f_{k+1}\right\|_{\infty} \leq 1+\varepsilon / 2, \quad f_{k+1}\left(x_{0}\right)=1
\end{aligned}
$$

and

$$
\left|f_{k+1}(x)\right|<\varepsilon / 2 \text { for all } x \text { in } X \backslash U_{k+1} .
$$

Let $n$ be a positive integer such that $1 / n<\varepsilon / 6$. We define

$$
f=\frac{2}{n} \sum_{k=1}^{n} f_{k}-1
$$

We have $f\left(x_{0}\right)=1$, and $|f(x)+1| \leq \varepsilon$ for $x \in X \backslash U$. To complete the proof, fix $x \in X \backslash U_{1}$ and denote by $k_{0}$ the greatest positive integer not greater than $n$ such 
that $x \in U_{k_{0}}$. We obtain

$$
\begin{aligned}
\left|f(x)+1-2 \frac{k_{0}-1}{n}\right| & =\left|\frac{2}{n}\left[\sum_{j=1}^{k_{0}-1}\left(f_{j}(x)-1\right)+f_{k_{0}}(x)+\sum_{j=k_{0}+1}^{n} f_{j}(x)\right]\right| \\
& \leq \frac{2}{n}\left[\left(k_{0}-1\right) \frac{\varepsilon}{2}+1+\varepsilon+\left(n-k_{0}\right) \frac{\varepsilon}{2}\right] \\
& =\frac{2}{n}+\frac{n+1}{n} \frac{\varepsilon}{2}<\varepsilon .
\end{aligned}
$$

Hence, we get $|\operatorname{Im} f(x)| \leq \varepsilon$ and $\|f\|_{\infty} \leq \varepsilon$.

To end the proof of this step, fix $\varepsilon>0$ and assume $T$ is discontinuous. There is an $f_{0} \in A$ such that $\left\|f_{0}\right\|_{\infty} \leq \varepsilon$ and $\left\|T f_{0}\right\|_{\infty}=1$. Since any function from $B$ achieves its sup-norm on $\mathrm{Ch} B$, we can assume there is a $y_{0} \in \mathrm{Ch} B$ with $T f_{0}\left(y_{0}\right)=1$. We put $U_{0}=\left\{y \in Y:\left|T f_{0}(y)-1\right|<\varepsilon\right\}$. By the lemma there is a $g \in B$ such that $\|g\| \leq 1+\varepsilon, g\left(y_{0}\right)=1,|g(y)+1|<\varepsilon$ for $y \in Y \backslash U$ and $|\operatorname{Im} g(y)|<\varepsilon$ for $y \in Y$. From (3) we get

$$
\tilde{\sigma}\left(T f_{0}\right)=\tilde{\sigma}\left(f_{0}\right)+K\left(\Delta f_{0}\right) \quad \text { and } \quad 1-\varepsilon \leq \Delta f<1
$$

hence,

$$
\operatorname{co}(K(-1,1-O(\varepsilon)) \cup\{2\}) \subset \tilde{\sigma}\left(g+T f_{0}\right) \subset \operatorname{co}(K(-1,1) \cup\{2\})+K(O(\varepsilon)) ;
$$

hence,

$$
c_{B}\left(g+T f_{0}, \pi / 2\right) \leq \sqrt{2}+O(\varepsilon) \text { and } c_{B}\left(g+T f_{0},-\pi / 2\right) \geq 1.6-O(\varepsilon),
$$

so

$$
c_{B}\left(g+T f_{0},-\pi / 2\right)-c_{B}\left(g+T f_{0}, \pi / 2\right) \geq 1.6-\sqrt{2}-O(\varepsilon) .
$$

Put $f_{1}=T^{-1} g$. Notice that for any $\varnothing \neq K \subset \mathbf{C}$ and any $c \geq 0$, we have $\rho(K+K(c)) \geq c$, so from (3) and the definition of $g$, we get $\Delta g \leq 2 \varepsilon$,

$$
\tilde{\sigma}(g)=\tilde{\sigma}\left(f_{1}\right)+K(\Delta g) \text { if } 0 \leq \Delta g \leq 2 \varepsilon,
$$

and

$$
\tilde{\sigma}\left(f_{1}\right)=\tilde{\sigma}(g)+K(-\Delta g) \text { if } \Delta g<0 .
$$

Hence, since $\left\|f_{0}\right\|_{\infty} \leq \varepsilon$, we get

$$
\left|c_{A}\left(f_{1}+f_{0}, \pm \pi / 2\right)-c_{A}\left(f_{1}, \pm \pi / 2\right)\right| \leq O(\varepsilon),
$$

and

$$
\left|c_{A}\left(f_{1}+f_{0},+\pi / 2\right)-c_{A}\left(f_{1}+f_{0},-\pi / 2\right)\right| \leq O(\varepsilon) .
$$

By (2) for any $f \in A$ the difference between $c_{A}(f, \varphi)$ and $c_{B}(T f, \varphi)$ does not depend on $\varphi$ so this gives a contradiction between (7) and (8).

Proof of the second step. Fix $f \in A$ with $\|f\|_{\infty}=1$. We can assume, as before, that there is an $x_{0} \in \mathrm{Ch} A$ such that $f\left(x_{0}\right)=1$. Put $U_{n}=\{x \in X: \mid f(x)-$ 1| $\left.<1 / n^{2}\right\}$, and let $g_{n} \in A$ be such that $\left\|g_{n}\right\|_{\infty} \leq 1+1 / n^{2}, g_{n}\left(x_{0}\right)=1$, and $\left|g_{n}(x)\right|<1 / n^{2}$ for $x \in X \backslash U_{n}$. We put $f_{n}=f+g_{n} / n$ and get

$$
1+1 / n \in \tilde{\sigma}\left(f_{n}\right) \subset \operatorname{co}(K(1) \cup\{1+1 / n\})+K\left(1 / n^{2}\right),
$$


and, hence, by a direct computation,

$$
\begin{aligned}
\rho\left(\tilde{\sigma}\left(f_{n}\right)\right) & \leq \rho\left(\tilde{\sigma}\left(f_{n}\right), 1+1 / n\right) \\
& \leq \rho\left(\operatorname{co}(K(1) \cup\{1+1 / n\})+K\left(1 / n^{2}\right), 1+1 / n\right) \\
& =1 / n+1 / n^{2}<2 / n .
\end{aligned}
$$

Therefore there is a sequence $\left(f_{n}\right)_{n=1}^{\infty}$ in $A$ which tends uniformly to $f$ such that $\rho\left(\tilde{\sigma}\left(f_{n}\right)\right) \rightarrow 0$.

Proof of the third step. For any $K \subset \mathbf{C}$ and any $c \geq 0$, we have $\rho(\operatorname{co}(K)+K(c)) \geq$ $c$ and, on the other hand, by (3), if $\Delta f<0$, then $\tilde{\sigma}(f)=\tilde{\sigma}(T f)+K(-\Delta f)$, so if $\Delta f<0$ then $\rho(\tilde{\sigma}(f)) \geq-\Delta f$, and this proves that $\Delta f \geq-\varepsilon$ for all $f \in \mathcal{A}_{\varepsilon}$.

\section{REFERENCES}

1. M. Cambern, Isometries of certain Banach algebras, Studia Math. 25 (1965), 217-225.

2. M. Cambern and V. Pathak, Isometries of spaces of differentiable functions, Math. Japan. 26 (1981), 253-260.

3. T. W. Gamelin, Uniform algebras, Prentice-Hall, Englewood Cliffs, N.J., 1969.

4. M. Nagasawa, Isomorphisms between commutative Banach algebras with an application to rings of analytic functions, Kōdai Math. Sem. Rep. 11 (1959), 182-188.

5. V. Pathak, Isometries of $C^{(n)}[0,1]$, Pacific J. Math. 94 (1981), 211-222.

6. _ Linear isometries of spaces of absolutely continuous functions, Canad. J. Math. 34 (1982), 298-306.

7. N. V. Rao and A. K. Roy, Linear isometries of some function spaces, Pacific J. Math. 38 (1971), 177-192.

Institute of MAThematics, WARSAW UNIVERSity, PKIN, 00-901 WARSAW, PolAND 